\title{
A Geometric Approach to Support Vector Machine (SVM) Classification
}

\author{
Michael E. Mavroforakis and Sergios Theodoridis, Senior Member, IEEE
}

\begin{abstract}
The geometric framework for the support vector machine (SVM) classification problem provides an intuitive ground for the understanding and the application of geometric optimization algorithms, leading to practical solutions of real world classification problems. In this work, the notion of "reduced convex hull" is employed and supported by a set of new theoretical results. These results allow existing geometric algorithms to be directly and practically applied to solve not only separable, but also nonseparable classification problems both accurately and efficiently. As a practical application of the new theoretical results, a known geometric algorithm has been employed and transformed accordingly to solve nonseparable problems successfully.
\end{abstract}

Index Terms-Classification, kernel methods, pattern recognition, reduced convex hulls, support vector machines (SVMs).

\section{INTRODUCTION}

$\mathbf{S}$ UPPORT vector machine (SVM) formulation of pattern recognition (binary) problems brings along a bunch of advantages over other approaches, e.g., [1] and [2], some of which are: 1) Assurance that once a solution has been reached, it is the unique (global) solution, 2) good generalization properties of the solution, 3) sound theoretical foundation based on learning theory [structural risk minimization (SRM)] and optimization theory, 4) common ground/formulation for the class separable and the class nonseparable problems (through the introduction of appropriate penalty factors of arbitrary degree in the optimization cost function) as well as for linear and nonlinear problems (through the so called "kernel trick") and, 5) clear geometric intuition on the classification task. Due to the above nice properties, SVM have been successfully used in a number of applications, e.g., [3]-[9].

The contribution of this work consists of the following. 1) It provides the theoretical background for the solution of the nonseparable (both linear and nonlinear) classification problems with linear (first degree) penalty factors, by means of the reduction of the size of the convex hulls of the training patterns. This task, although it is, in principle, of combinatorial complexity in nature, it is transformed to one of linear complexity by a series of theoretical results deduced and presented in this work. 2) It exploits the intrinsic geometric intuition to the full extent, i.e., not only theoretically but also practically (leading to an algorithmic solution), in the context of classification through the SVM approach. 3) It provides an easy way to relate each class with a different penalty factor, i.e., to relate each class

Manuscript received November 11, 2004; revised July 27, 2005.

The authors are with the Informatics and Telecommunications Department, University of Athens, Athens 15771, Greece (e-mail: mmavrof@di.uoa.gr; stheodor@di.uoa.gr).

Digital Object Identifier 10.1109/TNN.2006.873281 with a different risk (weight). 4) It applies a fast, simple, and easily conceivable algorithm to solve the SVM task. 5) It opens the road for applying other geometric algorithms, finding the closest pair of points between convex sets in Hilbert spaces, for the nonseparable SVM problem.

Although some authors have presented the theoretical background of the geometric properties of SVMs, exposed thoroughly in [10], the main stream of solving methods comes from the algebraic field (mainly decomposition). One of the best representative algebraic algorithms with respect to speed and ease of implementation, also presenting very good scalability properties, is the sequential minimal optimization (SMO) [11]. The geometric properties of learning [12] and specifically of SVMs in the feature space, have been pointed out early enough through the dual representation (i.e., the convexity of each class and finding the respective support hyperplanes that exhibit the maximal margin) for the separable case [13] and also for the nonseparable case through the notion of the reduced convex hull (RCH) [14]. However, the geometric algorithms presented until now [15], [16] are suitable only for solving directly the separable case. These geometric algorithms, in order to be useful, have been extended to solve indirectly the nonseparable case through the technique proposed in [17], which transforms the nonseparable problem to a separable one. However, this transformation (artificially extending the dimension of the input space by the number of training patterns) is equivalent to a quadratic penalty factor. Moreover, besides the increase of complexity due to the artificial expansion of the dimension of the input space, it has been reported that the generalization properties of the resulting SVMs can be poor [15].

The content of the rest of the paper has been structured as follows: In Section II, some preliminary material on SVM classification has been presented. In Section III, the notion of the reduced convex hull is defined and a direct and intuitive connection to the nonseparable SVM classification problem is presented. In the sequel, the main contribution of this work is displayed, i.e., a complete mathematical framework is devised to support the RCH and, therefore, make it directly applicable to practically solve the nonseparable SVM classification problem. Without this framework, the application of a geometric algorithm in order to solve the nonseparable case through $\mathrm{RCH}$ is practically impossible, since it is a problem of combinatorial complexity. In Section IV, a geometric algorithm is rewritten in the context of this framework, therefore, showing the practical benefits of the theoretical results derived herewith to support the RCH notion. Finally, in Section V, the results of the application of this algorithm to solve certain classification tasks are presented. 


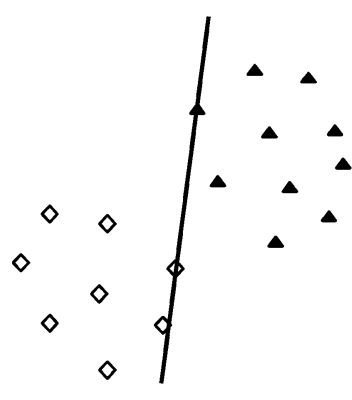

(a)

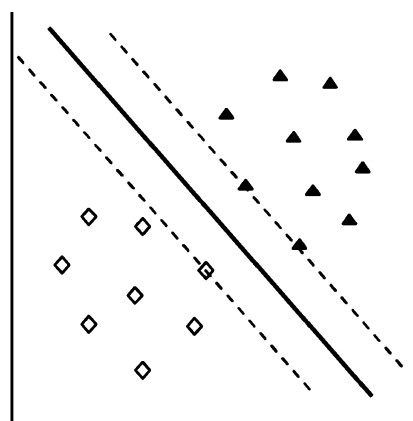

(b)
Fig. 1. Separating hyperplane exhibiting zero margin (a) compared to the maximal margin separating hyperplane, and (b) for the same classes of training samples presented in feature space.

\section{PRELIMINARY}

The complex and challenging task of (binary) classification or (binary) pattern recognition in supervised learning can be described as follows [18]: Given a set $\mathcal{X}$ of training objects (patterns) - each belonging to one of two classes-and their corresponding class identifiers, assign the correct class to a newly (not a member of $\mathcal{X}$ ) presented object; $(\mathcal{X}$ does not need any kind of structure except of being a nonempty set). For the task of learning, a measure of similarity between the objects of $\mathcal{X}$ is necessary, so that patterns of the same class are mapped "closer" to each other, as opposed to patterns belonging to different classes. A reasonable measure of similarity has the form $k: \mathcal{X} \times \mathcal{X} \rightarrow \mathbb{R},\left(x_{1}, x_{2}\right) \rightarrow k\left(x_{1}, x_{2}\right)$, where $k$ is (usually) a real (symmetric) function, called a kernel. An obvious candidate is the inner product $\left(x_{1} \mid x_{2}\right),{ }^{1}$ in case that $\mathcal{X}$ is an inner-product space (e.g., $\left.\mathbb{R}^{d}\right)$, since it leads directly to a measure of lengths through the norm derived from the inner product $\|x\|=\sqrt{(x \mid x)}$ and also to a measure of angles and hence to a measure of distances. When the set $\mathcal{X}$ is not an inner product space, it may be possible to map its elements $x$ to an inner product space, $\mathcal{H}$, through a (nonlinear) function $\Phi: \mathcal{X} \rightarrow \mathcal{H}$ such that $\mathbf{x} \equiv \Phi(x), \mathbf{x} \in \mathcal{H}, \forall x \in \mathcal{X}$. Under certain loose conditions (imposed by Mercer's theorem [19]), it is possible to relate the kernel function with the inner product of the feature space $\mathcal{H}$, i.e., $k\left(x_{1}, x_{2}\right)=\left(\Phi\left(x_{1}\right) \mid \Phi\left(x_{2}\right)\right)$ for all $x_{1}, x_{2} \in \mathcal{X}$. Then, $\mathcal{H}$ is known as a reproducing kernel Hilbert space (RKHS). RKHS is a very useful tool, because any Cauchy sequence converges to a limit in the space, which means that it is possible to approximate a solution (e.g., a point with maximum similarity) as accurately as needed.

\section{A. SVM Classification}

Simply stated, an SVM finds the best separating (maximal margin) hyperplane between the two classes of training samples in the feature space, as it is shown in Fig. 1.

A linear discriminant function has the form of the linear functional $f(x)=\langle w, x\rangle+c$, which corresponds to a hyperplane [20], dividing the feature space. If, for a given pattern mapped in the feature space to $x$, the value of $f(x)$ is a positive number,

\footnotetext{
${ }^{1}$ The notation $(x \mid y)$ will be used interchangeably with $\langle x, y\rangle$ for spaces which coincide with their dual.
}

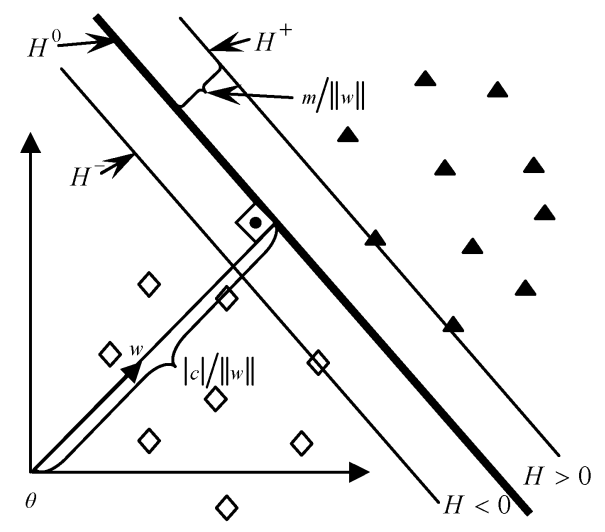

Fig. 2. Geometric interpretation of the maximal margin classification problem. Setting $H \equiv(\langle w, x\rangle /\|w\|)+(c /\|w\|)$ the hyperplanes $H^{0}: H=0, H^{-}$: $H=-(m /\|w\|)$ and $H^{+}: H=m /\|w\|$ are shown.

then the pattern belongs to the class labeled by the numeric value +1 ; otherwise, it belongs to the class with value -1 . Denoting as $y_{i}$ the numeric value of the class label of pattern $x_{i}$ and $m$ the maximum (functional) margin, the problem of classification is equivalent to finding the functional $f$ (satisfying $\left.y_{i}\left(\left\langle w, x_{i}\right\rangle+c\right) \geqslant m\right)$ that maximizes $m$.

In geometric terms, expressing the involved quantities in "lengths" of $w$ (i.e., $\|w\|)$, the problem is restated as follows: Find the hyperplane $H(w, c):\langle w, x\rangle+c=0$, maximizing the (geometric) margin $m /\|w\|$ and satisfying $y_{i}\left(\left(\left\langle w, x_{i}\right\rangle /\|w\|\right)+(c /\|w\|)\right)>m /\|w\|$ for all the training patterns.

The geometric margin $m /\|w\|$ represents the minimum distance of the training patterns of both classes from the separating hyperplane defined by $(w, c)$. The resulting hyperplane is called the maximal margin hyperplane. If the quantity $m /\|w\|$ is positive, then the problem is a linearly separable one. This situation is shown in Fig. 2.

It is clear that $H(w, c)=H(s w, s c), s>0$ (because of the linearity of inner product) and since $\|s w\|=|s|\|w\|$, a scaling of the parameters $w, c$ and $m$ does not change the geometry. Therefore, assuming $m=1$ (canonical hyperplane), the classification problem takes the equivalent form: Find the hyperplane

$$
H(w, c):\langle w, x\rangle+c=0
$$

maximizing the total (interclass) margin $2 /\|w\|$, or equivalently minimizing the quantity

$$
(1 / 2)\|w\|^{2}
$$

and satisfying

$$
y_{i}\left(\left\langle w, x_{i}\right\rangle+c\right) \geqslant 1
$$

This is a quadratic optimization problem (if the Euclidean norm is adopted) with linear inequality constraints and the standard algebraic approach is to solve the equivalent problem of minimizing the Lagrangian

$$
\mathcal{L}_{P} \equiv \frac{1}{2}\langle w, w\rangle-\sum_{i} a_{i}\left(y_{i}\left(\left\langle w, x_{i}\right\rangle+c\right)-1\right)
$$


subject to the constraints $a_{i} \geqslant 0$. The corresponding dual optimization problem is to maximize

$$
\mathcal{L}_{D} \equiv \sum_{i} a_{i}-\frac{1}{2} \sum_{i} \sum_{j} y_{i} y_{j} a_{i} a_{j}\left\langle x_{i}, x_{j}\right\rangle
$$

subject to the constraints

$$
a_{i} \geqslant 0
$$

and

$$
\sum_{i} y_{i} a_{i}=0 .
$$

Denote, for convenience, by $I^{-}$and $I^{+}$the sets of indices $i$, such that $y_{i}=-1$ and $y_{i}=+1$, respectively, and by $I$ the set of all indices, i.e., $I=I^{-} \cup I^{+}$.

The Karush-Kuhn-Tucker (KKT) optimality conditions provide the necessary and sufficient conditions that the unique solution has been found to the last optimization problem, i.e., (besides the initial constraints)

$$
\begin{aligned}
w & =\sum_{i} y_{i} a_{i} x_{i}=\sum_{i \in I^{+}} a_{i} x_{i}-\sum_{i \in I^{-}} a_{i} x_{i} \\
\sum_{i} y_{i} a_{i} & =0 \Leftrightarrow \sum_{i \in I^{+}} a_{i}=\sum_{i \in I^{-}} a_{i}
\end{aligned}
$$

and the KKT complementarity condition

$$
a_{i}\left(y_{i}\left(\left\langle w, x_{i}\right\rangle+c\right)-1\right)=0
$$

which means that, for the inactive constraints there is $a_{i}=0$ and for the active ones (when $y_{i}\left(\left\langle w, x_{i}\right\rangle+c\right)-1=0$ is satisfied) there is $a_{i} \geqslant 0$. The points with $a_{i}>0$ lie on the canonical hyperplane and are called support vectors. The interpretation of the KKT conditions [especially (8) and (9) with the extra reasonable nonrestrictive assumption that $\left.\sum_{i \in I^{+}} a_{i}=\sum_{i \in I^{-}} a_{i}=1\right]$ is very intuitive [1] and leads to the conclusion that the solution of the linearly separable classification problem is equivalent to finding the points of the two convex hulls [21] (each generated by the training patterns of each class) which are closest to each other and the maximum margin hyperplane a) bisects, and b) is normal to the line segment joining these two closest points, as seen in Fig. 3. The formal proof of this is presented in [13].

To address the (most common in real world applications) case of linearly nonseparable classification problem, for which any effort to find a separating hyperplane is hopeless, the only way for someone to reach a solution is to relax the data constraints. This is accomplished through the addition of margin slack variables $\xi_{i}$, which allow a controlled violation of the constraints [22]. Therefore, the constraints in (3) become

$$
y_{i}\left(\left\langle w, x_{i}\right\rangle+c\right) \geqslant 1-\xi_{i}
$$

where $\xi_{i} \geqslant 0$. It is clear that if $\xi_{i}>1$, then the point $x_{i}$ is misclassified by the hyperplane $H(w, c)$. The quantity $\xi_{i} /\|w\|$ has a clear geometric meaning: It is the distance of the point $x_{i}$ (in lengths of $w$ ) from the supporting hyperplane of its corresponding class; since $\xi_{i}$ is positive, $x_{i}$ lies in the opposite direction of the supporting hyperplane of its class, i.e., the corresponding supporting hyperplane separates $x_{i}$ from its own class. A natural way to incorporate the cost for the errors in classification is to augment the cost function (2) by the term $C \sum_{i} \xi_{i}$

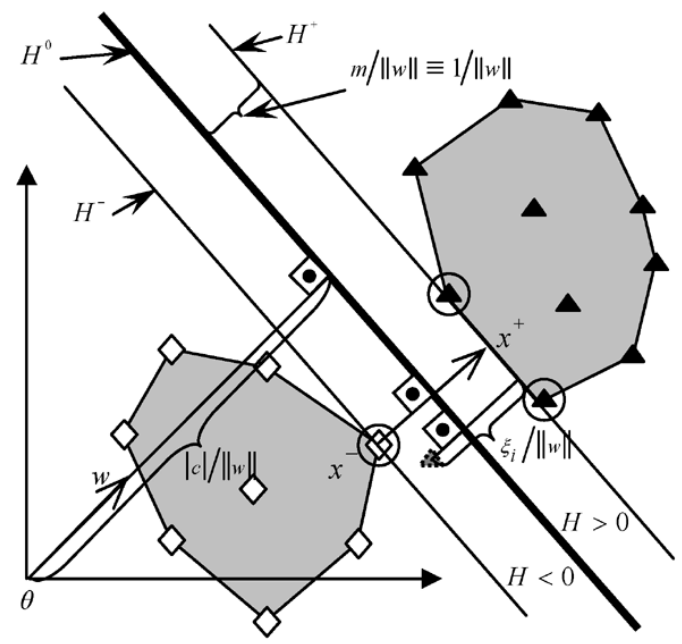

Fig. 3. Geometric interpretation of the maximal margin classification problem. Closest points are denoted by circles.

(although terms of the form $C \sum_{i} \xi_{i}^{d}$ have also been proposed), where $C$ is a free parameter (known also as regularization parameter or penalty factor) indicating the penalty imposed to the "outliers," i.e., higher value of $C$ corresponds to higher penalty for the "outliers" [23]. Therefore, the cost function (2) for the nonseparable case becomes

$$
\frac{1}{2}\|w\|^{2}+C \sum_{i} \xi_{i}
$$

Consequently, the Langrangian of the primal problem is

$$
\begin{aligned}
\mathcal{L}_{P} \equiv & \frac{1}{2}\langle w, w\rangle+C \sum_{i} \xi_{i} \\
& -\sum_{i} a_{i}\left(y_{i}\left(\left\langle w, x_{i}\right\rangle+c\right)-1+\xi_{i}\right)-\sum_{i} \nu_{i} \xi_{i}
\end{aligned}
$$

subject to the constraints $a_{i} \geqslant 0$ and $\nu_{i} \geqslant 0$ (introduced to ensure positivity of $\xi_{i}$ ). The corresponding dual optimization problem has again the form of (5), i.e., to maximize

$$
\mathcal{L}_{D} \equiv \sum_{i} a_{i}-\frac{1}{2} \sum_{i} \sum_{j} y_{i} y_{j} a_{i} a_{j}\left\langle x_{i}, x_{j}\right\rangle
$$

but now subject to the constraints

$$
0 \leqslant a_{i} \leqslant C
$$

and

$$
\sum_{i} y_{i} a_{i}=0 .
$$

It is interesting that neither the slack variables $\xi_{i}$ nor their associated Lagrange multipliers $\nu_{i}$ are present in the Wolfe dual formulation of the problem (a result of choosing $d=1$ as the exponent of the penalty terms) and that the only difference from the separable case is the impose of the upper bound $C$ to the Lagrange multipliers $a_{i}$.

However, the clear geometric intuition of the separable case has been lost; it is regained through the work presented in [14], [13] and [10], where the notion of the reduced convex hull, introduced and supported with new theoretical results in the next section, plays an important role. 


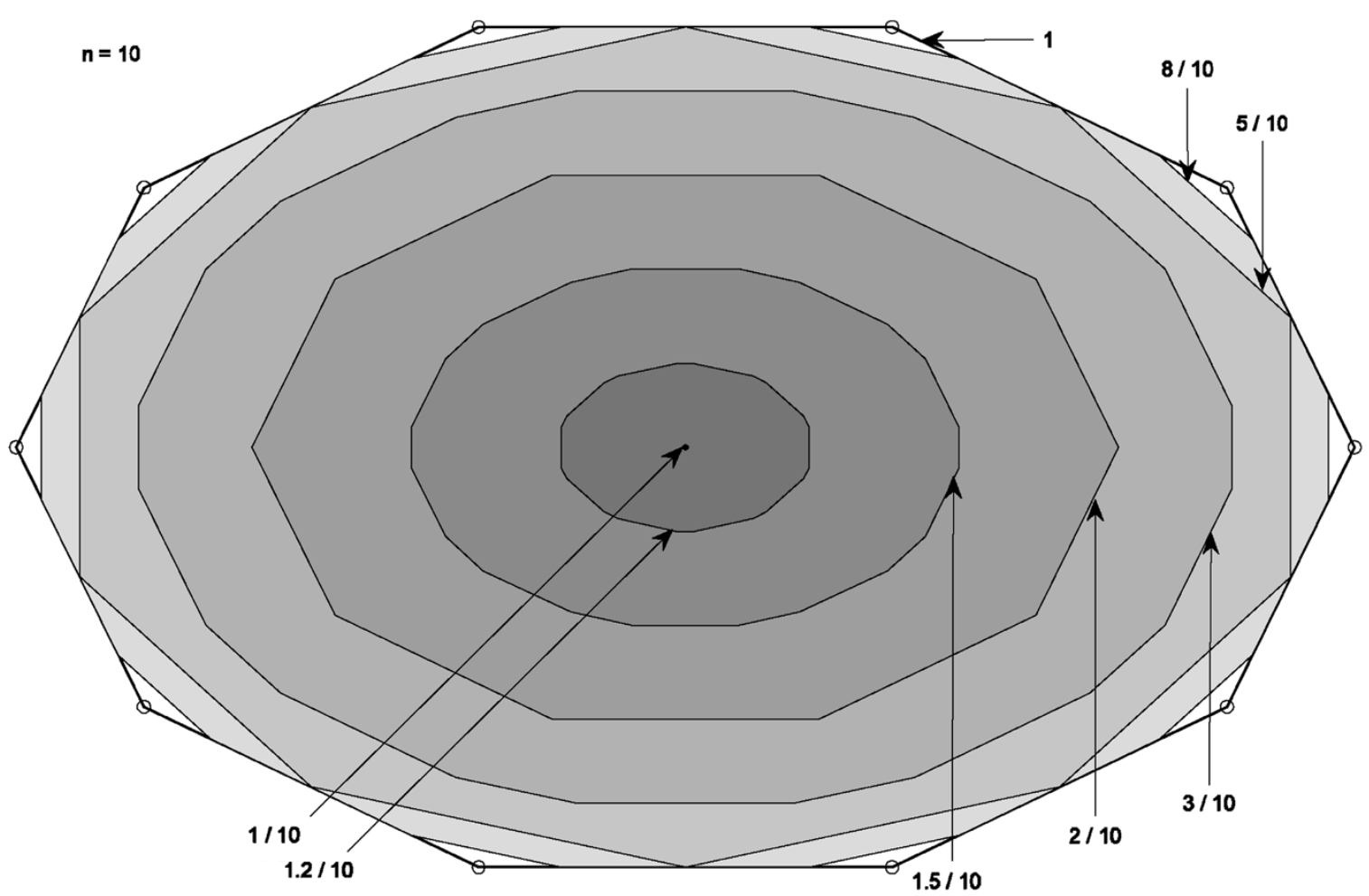

Fig. 4. Evolution of a convex hull with respect to $\mu$. (The corresponding $\mu$ of each RCH are the values indicated by the arrows.) The initial convex hull $(\mu=1)$, generated by ten points $(n=10)$, is successively reduced, setting $\mu$ to $8 / 10,5 / 10,3 / 10,2 / 10,1.5 / 10,1.2 / 10$ and finally $1 / 10$, which corresponds to the centroid. Each smaller (reduced) convex hull is shaded with a darker color.

\section{REDUCED CONVEX Hulls (RCH)}

The set of all convex combinations of points of some set $X$, with the additional constraint that each coefficient $a_{i}$ is upperbounded by a nonnegative number $\mu<1$, is called the reduced convex hull of $X$ and denoted by $\mathrm{R}(X, \mu)$

$$
\begin{aligned}
& R(X, \mu)=\left\{w: w=\sum_{i=1}^{k} a_{i} x_{i}, x_{i} \in X\right. \\
& \left.\qquad \sum_{i=1}^{k} a_{i}=1, \quad 0 \leqslant a_{i} \leqslant \mu\right\}
\end{aligned}
$$

Therefore, for the nonseparable classification task, the initially overlapping convex hulls, with a suitable selection of the bound $\mu$, can be reduced so that to become separable. Once separable, the theory and tools developed for the separable case can be readily applied. The algebraic proof is found in [14] and [13]; a totally geometric formulation of SVM leading to this conclusion is found in [10].

The effect of the value of bound $\mu$ to the size of the $\mathrm{RCH}$ is shown in Fig. 4.

In the sequel, we will prove some theorems and propositions that shed further intuition and usefulness to the $\mathrm{RCH}$ notion and at the same time form the basis for the development of the novel algorithm which is proposed in this paper.

Proposition 1: If all the coefficients $a_{i}$ of all the convex combinations forming the $\mathrm{RCH} \mathrm{R}(X, \mu)$ of a set $X$ with $k$ elements, are less than $1 / k$ (i.e., $\mu<1 / k$ ), then $\mathrm{R}(X, \mu)$ will be empty.

Proof: $a_{i}<1 / k \Rightarrow \sum_{i=1}^{k} a_{i}<\sum_{i=1}^{k}(1 / k)=$ $k(1 / k)=1 \Rightarrow \sum_{i=1}^{k} a_{i}<1$. Since $\sum_{i=1}^{k} a_{i}=1$ is needed to be true, it is clear that $\mathrm{R}(X, \mu)=\{\emptyset\}$.
Proposition 2: If for every $i$, there is $a_{i}=1 / k$ in a $\mathrm{RCH}$ $\mathrm{R}(X, \mu)$ of a set $X$ with $k$ different points as elements, then $\mathrm{R}(X, \mu)$ degenerates to a set of one single point, the centroid point (or barycenter) of $X$.

Proof: From the definition of the $\mathrm{RCH}$, it is

$$
\begin{aligned}
\mathrm{R}(X, \mu)= & \left\{w: w=\sum_{i=1}^{k} a_{i} x_{i}\right\} \\
= & \left\{w: w=(1 / k) \sum_{i=1}^{k} x_{i}\right\} \\
& =\{w: w=(1 / k) z\}, \\
& \text { where } z \equiv \sum_{i=1}^{k} x_{i} \text { is a single point. }
\end{aligned}
$$

Remark: It is clear that in an $\mathrm{RCH} \mathrm{R}(X, \mu)$, a choice of $\mu>1$ is equivalent with $\mu=1$ as the upper bound for all $a_{i}$, because it must be $\sum_{i=1}^{k} a_{i}=1$ and, therefore, $a_{i} \leqslant 1$. As a consequence of this and the above proposition, it is deduced that the RCH $\mathrm{R}(X, \mu)$ of a set $X$ will be either empty (if $\mu<1 / k)$, or grows from the centroid $(\mu=1 / k)$ to the convex hull $(\mu \geqslant 1)$ of $X$.

For the application of the above to real life algorithms, it is absolutely necessary to have a clue about the extreme points of the $\mathrm{RCH}$. In the case of the convex hull, generated by a set of points, only a subset of these points constitute the set of extreme points, which, in turn, is the minimal representation of the convex hull. Therefore, only a subset of the original points is needed to be examined and not every point of the convex hull [24]. In contrast, as it will soon be seen, for the case of $\mathrm{RCH}$, its extreme points are the result of combinations of the extreme points of 
the original convex hull, which, however, do not belong to the $\mathrm{RCH}$, as it was deduced above.

In the sequel, it will be shown that not any combination of the extreme points of the original convex hull leads to extreme points of the RCH, but only a small subset of them. This is the seed for the development of the novel efficient algorithm to be presented later in this paper.

Lemma 1: For any point $w \in \mathrm{R}(X, \mu)$, if there exists a reduced convex combination $w=\sum_{i=1}^{k} a_{i} x_{i}$, with $x_{i} \in X, \sum_{i=1}^{k} a_{i}=1,0 \leqslant a_{i} \leqslant \mu$ and at least one coefficient $a_{r}, 1 \leqslant r \leqslant k$, not belonging in the set $S=\{0, \quad 1-\lfloor 1 / \mu\rfloor \mu, \quad \mu\}$, where $\lfloor 1 / \mu\rfloor$ is the integer part of the ratio $1 / \mu$, then there exists at least another coefficient $a_{s}, 1 \leqslant s \leqslant k, r \neq s$, not belonging in the set $S$, i.e., there cannot be a reduced convex combination with just one coefficient not belonging in $S$.

Proof: The lengthy proof of this Lemma, is found in Appendix.

Theorem 1: The extreme points of an $\mathrm{RCH} \mathrm{R}(X, \mu)=$ $\left\{w: w=\sum_{i=1}^{k} a_{i} x_{i}, x_{i} \in X, \sum_{i=1}^{k} a_{i}=1,0 \leqslant a_{i} \leqslant \mu\right\}$

have coefficients $a_{i}$ belonging to the set $S=$ $\{0, \quad 1-\lfloor 1 / \mu\rfloor \mu, \quad \mu\}$.

Proof: In the case that $\mu=1$ the theorem is obviously true since $\mathrm{R}(X, 1)$ is the convex hull of $X$, i.e., $\mathrm{R}(X, 1)=\operatorname{conv} X$ and, therefore, all the extreme points belong to the set $X$. Hence, if $x_{i}$ is an extreme point, its $j$ th coefficient $a_{i, j}$ is

$$
a_{i, j}=\left\{\begin{array}{ll}
0, & i \neq j \\
1, & i=j
\end{array} .\right.
$$

For $0<\mu<1$ the theorem will be proved by contradiction: Assuming that a point $w \in \mathrm{R}(X, \mu)$ is an extreme point, with some coefficients not belonging in $S$, a couple of other points $w_{1}, w_{2} \in \mathrm{R}(X, \mu)$ are needed to be found and then to be proved that $w$ belongs to the line segment $\left[w_{1}, w_{2}\right]$. As two points are needed, two coefficients have to be found not belonging in $S$. However, this is the conclusion of Lemma 1, which ensures that, if there exists a coefficient of a reduced convex combination not belonging in $S$, there exists a second one not belonging in $S$ as well.

Therefore, let an extreme point $w \in \mathrm{R}(X, \mu)$, where $w=$ $\sum_{i=1}^{k} a_{i} x_{i}$, that have at least two coefficients $a_{r}$ and $a_{s}, r \neq s$, such that $0<a_{r}, a_{s}<\mu$ and $a_{r}, a_{s} \notin\{0,1-\lfloor 1 / \mu\rfloor \mu, \mu\}$. Let also $\delta>0$ such that $\delta<\min \left\{a_{r}, a_{s}\right\}$ and $\delta<\mu-$ $\max \left\{a_{r}, a_{s}\right\}$, i.e., it is $0 \leqslant a_{r} \pm \delta, a_{r} \pm \delta \leqslant \mu$. Consequently, the points $w_{1}, w_{2}$ are constructed as follows:

$$
w_{1}=\sum_{i \neq r, s} a_{i} x_{i}+\left(a_{r}+\delta\right) x_{r}+\left(a_{s}-\delta\right) x_{s}
$$

and

$$
w_{2}=\sum_{i \neq r, s} a_{i} x_{i}+\left(a_{r}-\delta\right) x_{r}+\left(a_{s}+\delta\right) x_{s} .
$$

For the middle point of the line segment $\left[w_{1}, w_{2}\right]$, it is $1 / 2 w_{1}+$ $1 / 2 w_{2}=\sum_{i \neq r, s} a_{i} x_{i}+1 / 2\left(a_{r}+\delta+a_{r}-\delta\right) x_{r}+1 / 2\left(a_{s}-\right.$ $\left.\delta+a_{s}+\delta\right) x_{s}=1 / 2\left(\sum_{i \neq r, s} a_{i} x_{i}+\left(a_{r}+\delta\right) x_{r}+\left(a_{s}-\delta\right) x_{s}\right)$ $+1 / 2\left(\sum_{i \neq r, s} a_{i} x_{i}+\left(a_{r}-\delta\right) x_{r}+\left(a_{s}+\delta\right) x_{s}\right)=\sum_{i=1}^{k} a_{i} x_{i}$ $=w$, which is a contradiction to the assumption that $w$ is an extreme point. This proves the theorem.
Proposition 3: Each of the extreme points of an $\mathrm{RCH}$

$$
\mathrm{R}(X, \mu)=\left\{w: w=\sum_{i=1}^{k} a_{i} x_{i}, x_{i} \in X, \sum_{i=1}^{k} a_{i}=1,0 \leqslant a_{i} \leqslant \mu\right\}
$$

is a reduced convex combination of $m=\lceil 1 / \mu\rceil$ (distinct) points of the original set $X$, where $\lceil 1 / \mu\rceil$ is the smallest integer for which it is $\lceil 1 / \mu\rceil \geqslant 1 / \mu$. Furthermore, if $1 / \mu=\lceil 1 / \mu\rceil$, then all $a_{i}=\mu$; otherwise, $a_{i}=\mu$ for $i=1, \ldots, m-1$ and $a_{m}=$ $1-\lfloor 1 / \mu\rfloor \mu$.

Proof: Theorem 1 states that the only coefficients through which a point from the original set $X$ contributes to an extreme point of the $\operatorname{RCH} \mathrm{R}(X, \mu)$ are either $\mu$ or $1-\lfloor 1 / \mu\rfloor \mu$.

If $\lfloor 1 / \mu\rfloor=1 / \mu$, then $1-\lfloor 1 / \mu\rfloor \mu=0$; hence, the only coefficient valid is $\mu$ and, since $\sum_{i} a_{i}=1$ and $a_{i}=\mu$, it is $m \mu=1 \Leftrightarrow m=1 / \mu=\lceil 1 / \mu\rceil$.

If $(1 / \mu)-\lfloor 1 / \mu\rfloor \equiv \rho$ with $0<\rho<1$, then $1-\lfloor 1 / \mu\rfloor \mu=\mu \rho$ and, therefore, $1-\lfloor 1 / \mu\rfloor \mu<\mu$. Let $w$ be an extreme point of $\mathrm{R}(X, \mu), q$ be the number of points contributing to $w$ with coefficient $\mu$ and $p$ the number of points with coefficient

$$
1-\lfloor 1 / \mu\rfloor \mu, \quad \text { i.e., } w=\mu \sum_{i=1}^{q} x_{i}+(1-\lfloor 1 / \mu\rfloor \mu) \sum_{j=1}^{p} x_{j} .
$$

Since

$$
\sum_{i} a_{i}=1
$$

there is

$$
\begin{aligned}
& p(1-\lfloor 1 / \mu\rfloor \mu)+q \mu \\
& \quad=1 \Rightarrow p(1 / \mu)-p\lfloor 1 / \mu\rfloor+q=1 / \mu .
\end{aligned}
$$

If $p=1$, then (18) becomes $(1 / \mu)-\lfloor 1 / \mu\rfloor+q=1 / \mu \Rightarrow q=$ $\lfloor 1 / \mu\rfloor$; hence, $m=p+q=1+\lfloor 1 / \mu\rfloor=\lceil 1 / \mu\rceil$ which is the desired result.

Therefore, the remaining case is when $p>1$. Assuming that there exist at least two initial points $x_{u}$ and $x_{v}$ with coefficient $1-\lfloor 1 / \mu\rfloor \mu$, the validity of the proposition will be proved by contradiction. Since it is true $0<1-\lfloor 1 / \mu\rfloor \mu<\mu$ for this case, there exists a real positive number $\delta>0$ s.t. $\delta<$ $\min (1-\lfloor 1 / \mu\rfloor \mu, \mu-(1-\lfloor 1 / \mu\rfloor \mu))$. Let $a_{r}=1-\lfloor 1 / \mu\rfloor \mu-\delta$ and $a_{s}=1-\lfloor 1 / \mu\rfloor \mu+\delta$; using them, let $w_{1}=\mu \sum_{i=1}^{q} x_{i}+$ $(1-\lfloor 1 / \mu\rfloor \mu) \sum_{j=1}^{p-2} x_{j}+a_{r} x_{u}+a_{s} x_{v}$ and $w_{2}=\mu \sum_{i=1}^{q} x_{i}+$ $(1-\lfloor 1 / \mu\rfloor \mu) \sum_{j=1}^{p-2} x_{j}+a_{s} x_{u}+a_{r} x_{v}$. Obviously, since $0<$ $a_{r}, a_{s}<\mu$, the points $w_{1}$ and $w_{2}$ belong in the $\mathrm{RCH} \mathrm{R}(X, \mu)$. Taking into consideration that $a_{r}+a_{s}=1-\lfloor 1 / \mu\rfloor \mu-\delta+$ $1-\lfloor 1 / \mu\rfloor \mu+\delta=2(1-\lfloor 1 / \mu\rfloor \mu)$, the middle point of the line segment $\left[w_{1}, w_{2}\right]$ is $(1 / 2) w_{1}+(1 / 2) w_{2}=\mu \sum_{i=1}^{q} x_{i}+(1-$ $\lfloor 1 / \mu\rfloor \mu) \sum_{j=1}^{p-2} x_{j}+\left(\left(a_{r}+a_{2}\right) / 2\right)\left(x_{u}+x_{v}\right)=\mu \sum_{i=1}^{q} x_{i}+$ $(1-\lfloor 1 / \mu\rfloor \mu) \sum_{j=1}^{p} x_{j}=w$. Therefore, $w$ cannot be the extreme point of the RCH $\mathrm{R}(X, \mu)$, which contradicts with the assumption that $p>1$. This concludes the proof.

Remark: For the coefficients $\lambda \equiv 1-\lfloor 1 / \mu\rfloor \mu$ and $\mu$, it holds $0 \leqslant \lambda<\mu$. This is a byproduct of the proof of the above Proposition 3. 
Remark: The separation hyperplane depends on the pair of closest points of the convex hulls of the patterns of each class, and each such point is a convex combination of some extreme points of the RCHs. As, according to the above Theorem, each extreme point of the RCHs depends on $\lceil 1 / \mu\rceil$ original points (training patterns), it follows directly that the number of support vectors (points with nonzero Lagrange multipliers) is at least $\lceil 1 / \mu\rceil$, i.e, the lower bound of the number of initial points contributing to the discrimination function is $\lceil 1 / \mu\rceil$ (Fig. 5).

Remark: Although the above Theorem 1, along with Proposition 3 , restrict considerably the candidates to be extreme points of the $\mathrm{RCH}$, since they should be reduced convex combinations of $\lceil 1 / \mu\rceil$ original points and also with specific coefficients (belonging to the set $S$ ), the problem is still of combinatorial nature, because each extreme point is a combination of $\lceil 1 / \mu\rceil$ out of $k$ initial points for each class. This is shown in Fig. 5. Theorem 1 provides the necessary but not sufficient condition for a point to be extreme in an $\mathrm{RCH}$. The set of points satisfying the condition is larger than the set of extreme points; these are the "candidate to be extreme points," shown in Fig. 5. Therefore, the solution of the problem of finding the closest pair of points of the two reduced convex hulls essentially entails the following three stages:

1) identifying all the extreme points of each of the RCHs, which are actually subsets of the candidates to be extreme points pointed out by Theorem 1;

2) finding the subsets of the extreme points that contribute to the closest points, one for each set;

3) determining the specific convex combination of each subset of the extreme points for each set, which gives each of the two closest points.

However, in the algorithm proposed herewith, it is not the extreme points themselves that are needed, but their inner products (projections onto a specific direction). This case can be significantly simplified through the next theorem.

Lemma 2: Let $S=\left\{s_{i} \mid s_{i} \in \mathbb{R}, i=1, \ldots, n\right\}, \lambda \geqslant 0, \mu>$ 0 , and $\lambda \neq \mu$, with $k \mu+\lambda=1$. The minimum weighted sum on $S$ (for $k$ elements of $S$ if $\lambda=0$, or $k+1$ elements of $S$ if $\lambda>0$ ) is the expression $\lambda s_{i_{1}}+\mu \sum_{j=2}^{k+1} s_{i_{j}}$, if $0<\mu<\lambda$, or $\mu \sum_{j=1}^{k} s_{i_{j}}+\lambda s_{i_{k+1}}$, if $0<\lambda<\mu$, or $\mu \sum_{j=1}^{k} s_{i_{j}}$, if $\lambda=0$, where $s_{i_{p}} \leqslant s_{i_{q}}$, if $p<q$.

Proof: The proof of this Lemma is found in the Appendix.

Theorem 2: The minimum projection of the extreme points of an $\mathrm{RCH}$

$\mathrm{R}(X, \mu)=\left\{w: w=\sum_{i=1}^{k} a_{i} x_{i}, x_{i} \in X, \sum_{i=1}^{k} a_{i}=1,0 \leqslant a_{i} \leqslant \mu\right\}$

in the direction $p$ (setting $\lambda=1-\lfloor 1 / \mu\rfloor \mu$ and $m=\lfloor 1 / \mu\rfloor$ ) is

- $\mu \sum_{i=1}^{m} s_{i_{j}}$, if $0<\mu$ and $\lambda=0$;

- $\mu \sum_{j=1}^{m} s_{i_{j}}+\lambda s_{i_{m+1}}$, if $0<\lambda<\mu$;

where $s_{i_{j}}=\left(p \mid x_{j}\right) /\|p\|$ and $s_{i}$ is an ordering, such that $s_{i_{p}} \leqslant$ $s_{i_{q}}$ if $p<q$.

Proof: The extreme points of $\mathrm{R}(X, \mu)$ are of the form $z_{i}=\sum_{j=1}^{k} \alpha_{j} x_{j}$, where $k=|X|, x_{j} \in X, \sum_{j=1}^{k} \alpha_{j}=1$,
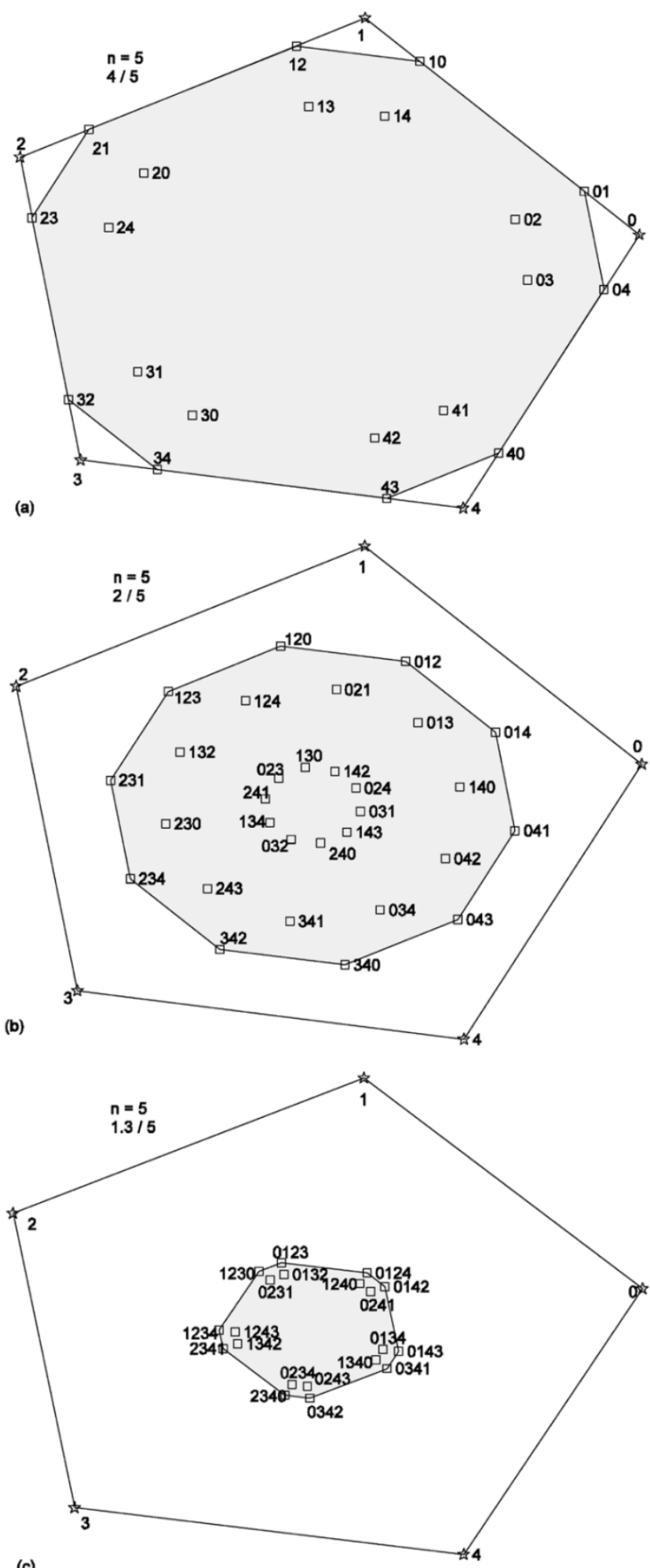

(c)

Fig. 5. Three RCHs, (a) R(P 5, 4/5), ${ }^{2}$ (b) R(P $\left.5,2 / 5\right)$, and (c) R(P 5, 1.3/5), are shown, generated by five points (stars), to present the points that are candidates to be extreme, marked by small squares. Each candidate to be extreme point in the $\mathrm{RCH}$ is labeled so as to present the original points from which it has been constructed, i.e., point (01) results from points (0) and (1); the last label is the one with the smallest coefficient.

and $\alpha_{j} \in\{0, \quad 1-\lfloor 1 / \mu\rfloor \mu, \quad \mu\}$. Therefore, taking into account that if $\lambda>0$, it is always $\lambda<\mu$, as it follows from the

${ }^{2} \mathrm{P} n$ stands for a (convex) Polygon of $n$ vertices. 


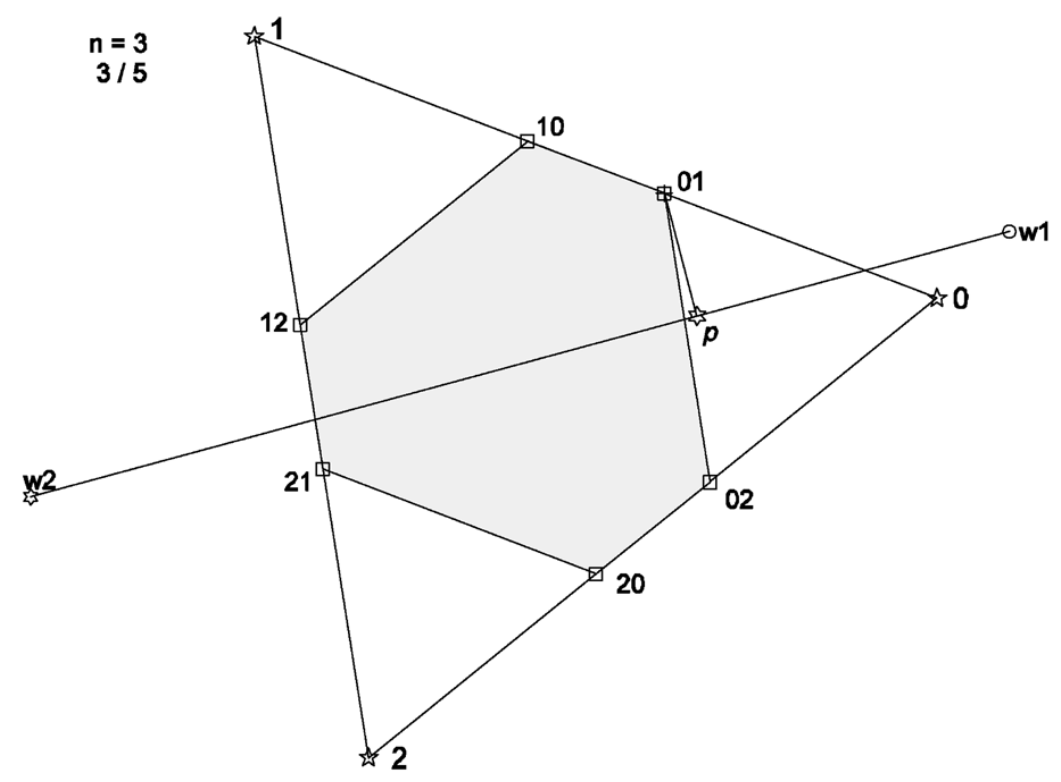

Fig. 6. Minimum projection $p$ of the RCH R(P3,3/5), generated by three points and having $\mu=3 / 5$, onto the direction $w_{2}-w_{1}$ belongs to the point $(01)$, which is calculated, according to Theorem 2 , as the ordered weighted sum of the projection of only $\lceil 5 / 3\rceil=2$ points $[(0)$ and (1)] of the three initial points. The magnitude of the projection, in lengths of $\left\|w_{2}-w_{1}\right\|$ is $(3 / 5)\left(x_{0} \mid w_{2}-w_{1}\right)+(2 / 5)\left(x_{1} \mid w_{2}-w_{1}\right)$.

Corollary of Proposition 3, the projection of an extreme point has the form

$$
\left(p \mid z_{i}\right) /\|p\|=\left(p \mid \sum_{j=1}^{k} \alpha_{j} x_{j}\right)=\sum_{j=1}^{k} \alpha_{j}\left(p \mid x_{j}\right)=\sum_{j=1}^{k} \alpha_{j} s_{\iota_{j}}
$$

and, according to the above Lemma 2, proves the theorem.

Remark: In other words, the previous Theorem states that the calculation of the minimum projection of the $\mathrm{RCH}$ onto a specific direction does not need the direct formation of all the possible extreme points of $\mathrm{RCH}$, but only the calculation of the projections of the $n$ original points and then the summation of the first least $\lceil 1 / \mu\rceil$ of them, each multiplied with the corresponding coefficient imposed by Theorem 2 . This is illustrated in Fig. 6.

Summarizing, the computation of the minimum projection of an $\mathrm{RCH}$ onto a given direction, entails the following steps:

1) compute the projections of all the points of the original set;

2) sort the projections in ascending order;

3) select the first (smaller) $\lceil 1 / \mu\rceil$ projections;

4) compute the weighted average of these projections, with weights suggested in Theorem 2.

Proposition 4: A linearly nonseparable SVM problem can be transformed to a linearly separable one through the use of $\mathrm{RCHs}$ (by a suitable selection of the reduction factor $\mu$ for each class) if and only if the centroids of the classes do not coincide. in [14].

\section{GEOMETRIC AlgORIthM FOR SVM SEPARABLE AND NONSEPARABLE TASKS}

As it has already been pointed out, an iterative, geometric algorithm for solving the linearly separable SVM problem has been presented recently in [16]. This algorithm, initially proposed by Kozinec for finding a separating hyperplane and improved by Schlesinger for finding an $\varepsilon$-optimal separating hyperplane, can be described by the following three steps (found and explained in [16], reproduced here for completeness).

1) Initialization: Set the vector $w_{1}$ to any vector $x \in X_{1}$ and $w_{2}$ to any vector $x \in X_{2}$.

2) Stopping Condition: Find the vector $x_{t}$ closest to the hyperplane as $x_{t}=\arg \min _{i \in I_{1} \cup I_{2}} m\left(x_{i}\right)$ where

$$
m\left(x_{i}\right)= \begin{cases}\frac{\left\langle x_{i}-w_{2}, w_{1}-w_{2}\right\rangle}{\left\|w_{1}-w_{2} \mid\right\|}, & \text { for } i \in I_{1} \\ \left\langle\frac{\left.x_{i}-w_{1}, w_{2}-w_{1}\right\rangle}{\left\|w_{1}-w_{2}\right\|},\right. & \text { for } i \in I_{2}\end{cases}
$$

If the $\varepsilon$-optimality condition $\left\|w_{1}-w_{2}\right\|-m\left(x_{t}\right)<\varepsilon$ holds, then the vector $w=w_{1}-w_{2}$ and $b=$ $1 / 2\left(\left\|w_{1}\right\|^{2}-\left\|w_{2}\right\|^{2}\right)$ defines the $\varepsilon$-solution; otherwise, go to step 3).

3) Adaptation: If $x_{t} \in X_{1}$ set $w_{2}^{\text {new }}=w_{2}$ and compute $w_{t}^{\text {new }}=(1-q) w_{1}+q x_{t}$, where $q=\min \left(1,\left(\left\langle w_{1}-w_{2}, w_{1}-x_{t}\right\rangle\right) /\left(\left\|w_{1}-x_{t}\right\|^{2}\right)\right) ;$ otherwise, set $w_{1}^{\text {new }}=w_{1}$ and compute $w_{2}^{\text {new }}=(1-q) w_{2}+q x_{t}$, where $q=\min \left(1,\left(\left\langle w_{2}-w_{1}, w_{2}-x_{t}\right\rangle\right) /\left(\left\|w_{2}-x_{t}\right\|^{2}\right)\right)$. Continue with step 2).

The above algorithm, which is shown in work schematically in Fig. 7, is easily adapted to be expressed through the kernel function of the input space patterns, since the vectors of the feature space are present in the calculations only through norms 


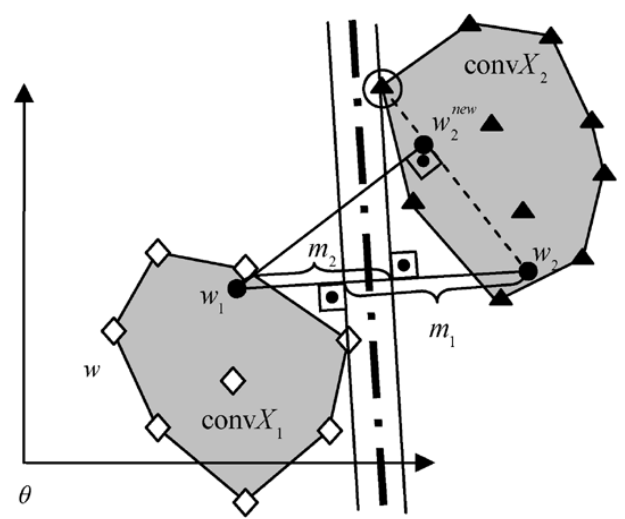

Fig. 7. Quantities, involved in S-K algorithm, are shown here for simplicity for (not reduced) convex hulls: $w_{1}$ is the best (until current step) approximation to the closest point of conv $X_{1}$ to conv $X_{2} ; m_{2}$ is the distance of $w_{1}$ from the closest projection of points of $X_{2}$ onto $\left(w_{1}-w_{2}\right)$ in lengths of $\left(w_{1}-w_{2}\right)$. The new $w$ belongs to the set with the least $m$ (e.g., in this case in conv $X_{2}$ ) and it is the closest point of the line segment with one end the old $w$ and the other end the point presenting the closest projection $\left(m_{2}\right)$, which in the figure is circled; this new $w$ is shown in the figure as $w_{2}^{\text {new }}$.

and inner products. Besides, a caching scheme can be applied with only $O\left(\left|I_{1}\right|+\left|I_{2}\right|\right)$ storage requirements.

The adaptation of the above algorithm is easy, with the mathematical toolbox for RCHs presented above and after making the following observations.

1) $w_{1}$ and $w_{2}$ should be initialized in such a way that it is certain they belong to the RCHs of $X_{1}$ and $X_{2}$, respectively. An easy solution is to use the centroid of each class as such. The algorithm secures that $w_{1}$ and $w_{2}$ evolve in such a way that they are always in their respective RCHs and converge to the nearest points.

2) Instead of the initial points (i.e., $x \in X_{1} \cup X_{2}$ ), all the candidates to be extreme points of the $\mathrm{RCH}$ have to be examined. However, actually what matters is not the absolute position of each extreme point but their projection onto $w_{1}-w_{2}$ or to $w_{2}-w_{1}$, if the points to be examined belong to the RCHs of $X_{1}$ and $X_{2}$, respectively.

3) The minimum projection belongs to the point which is formed according to Theorem 2.

According to the above, and for the clarity of the adapted algorithm to be presented, it will be helpful that some definitions and calculations of the quantities involved are provided beforehand.

At each step, the points $w_{1}$ and $w_{2}$, representing the closest points (up to that step) for each class respectively, are known through the coefficients $a_{i}$, i.e., $w_{1}=\sum_{i \in I_{1}} a_{i} x_{i}$ and $w_{2}=$ $\sum_{i \in I_{2}} a_{i} x_{i}$. However, the calculations do not involve $w_{1}$ and $w_{2}$ directly, but only through inner products, which is also true for all points. This is expected, since the goal is to compare distances and calculate projections and not to examine absolute positions. This is the point where the "kernel trick" comes into the scene, allowing the transformation of the linear to a nonlinear classifier.

The aim at each step is to find the point $z_{r}$, belonging to any of the RCHs of both classes, which minimizes the margin $m\left(z_{r}\right)$, defined [as in (19)] as

$$
m\left(z_{r}\right)=\left\{\begin{array}{ll}
\frac{\left\langle z_{1 r}-w_{2}, w_{1}-w_{2}\right\rangle}{\left\|w_{1}-w_{2}\right\|}, & z_{1 r} \in \mathrm{R}\left(X_{1}, \mu_{1}\right) \\
\frac{\left\langle z_{2 r}-w_{1}, w_{2}-w_{1}\right\rangle}{\left\|w_{1}-w_{2}\right\|}, & z_{2 r} \in \mathrm{R}\left(X_{2}, \mu_{2}\right)
\end{array} .\right.
$$

The quantity $m\left(z_{r}\right)$ is actually the distance, in lengths of $\left\|w_{1}-w_{2}\right\|$, of one of the closest points $\left(w_{1}\right.$ or $\left.w_{2}\right)$ from the closest projection of the $\mathrm{RCH}$ of the other class, onto the line defined by the points $w_{1}$ and $w_{2}$. This geometric interpretation is clearly shown in Fig. 7. The intermediate calculations, required for (20), are given in the Appendix.

According to the above, the algorithm becomes

\section{1) Initialization:}

a) Set $\lambda_{1} \equiv 1-\left\lfloor 1 / \mu_{1}\right\rfloor \mu_{1}, m_{1} \equiv\left\lfloor 1 / \mu_{1}\right\rfloor, \lambda_{2} \equiv 1-$ $\left\lfloor 1 / \mu_{2}\right\rfloor \mu_{2}$, and $m_{2} \equiv\left\lfloor 1 / \mu_{2}\right\rfloor$ and secure that $\mu_{1} \geqslant$ $1 /\left|I_{1}\right|$ and $\mu_{2} \geqslant 1 /\left|I_{2}\right|$.

b) Set the vectors $w_{1}$ and $w_{2}$ to be the centroids of the corresponding convex hulls, i.e., set $a_{i}=1 /\left|I_{1}\right|, i \in$ $I_{1}$ and $a_{i}=1 /\left|I_{2}\right|, i \in I_{2}$.

2) Stopping condition: Find the vector

$z_{r}=\left\{\begin{array}{lll}z_{1 r}=\sum_{i \in I_{1}} b_{i} x_{i}, & b_{i} \in\left\{0, \lambda_{1}, \mu_{1}\right\}, & \sum_{i \in I_{1}} b_{i}=1 \\ z_{2 r}=\sum_{i \in I_{2}} b_{i} x_{i}, & b_{i} \in\left\{0, \lambda_{2}, \mu_{2}\right\}, & \sum_{i \in I_{2}} b_{i}=1\end{array}\right.$

(actually the coefficients $b_{i}$ ) $\quad$ s.t. $z_{r}=$ $\arg \min _{z_{1 r} \in \mathrm{R}\left(X_{1}, \mu_{1}\right), z_{2 r} \in \mathrm{R}\left(X_{2}, \mu_{2}\right)}\left(m\left(z_{1 r}\right), m\left(z_{2 r}\right)\right)$

where

$m\left(z_{r}\right)= \begin{cases}\frac{\left\langle z_{1 r}-w_{2}, w_{1}-w_{2}\right\rangle}{\left\|w_{1}-w_{2}\right\|}, & z_{1 r} \in \mathrm{R}\left(X_{1}, \mu_{1}\right) \\ \frac{\left\langle z_{2 r}-w_{1}, w_{2}-w_{1}\right\rangle}{\left\|w_{1}-w_{2}\right\|}, & z_{2 r} \in \mathrm{R}\left(X_{2}, \mu_{2}\right)\end{cases}$

using (53) and (54).

If the $\varepsilon$-optimality condition $\left\|w_{1}-w_{2}\right\|-m\left(z_{r}\right)<\varepsilon$ [calculated after (44), (53) and (54)] holds, then the vector $w=w_{1}-w_{2}$ and $c=1 / 2\left(\left\|w_{1}\right\|^{2}-\left\|w_{2}\right\|^{2}\right)$ defines the $\varepsilon$-solution; otherwise, go to step 3$)$.

3) Adaptation: If $z_{r}=z_{1 r} \in \mathrm{R}\left(X_{1}, \mu_{1}\right)$, set $w_{2}^{\text {new }}=w_{2}$ and compute $w_{1}^{\text {new }}=q_{1} z_{1 r}+\left(1-q_{1}\right) w_{1}$, where

$q_{1}=\min \left(1,\left(\left\langle w_{1}-w_{2}, w_{1}-z_{1 r}\right\rangle\right) /\left(\left\|w_{1}-z_{1 r}\right\|^{2}\right)\right)$

and

$$
\begin{array}{r}
\left(\left\langle w_{1}-w_{2}, w_{1}-z_{1 r}\right\rangle\right) /\left(\left\|w_{1}-z_{1 r}\right\|^{2}\right) \\
=\left(A-\left\langle w_{1}, z_{1 r}\right\rangle-C+\left\langle w_{2}, z_{1 r}\right\rangle\right) / \\
\left(A+\left\langle z_{1 r}, z_{1 r}\right\rangle-2\left\langle w_{1}, z_{1 r}\right\rangle\right)
\end{array}
$$

[using (57)-(59)]; hence $a_{i}^{\text {new }}=q_{1} b_{i}+\left(1-q_{1}\right) a_{i}, i \in$ $I_{1}$; otherwise, set $w_{1}^{\text {new }}=w_{1}$ and compute $w_{2}^{\text {new }}=$ $q z_{2 r}+(1-q) w_{2}$, where

$q_{2}=\min \left(1,\left(\left\langle w_{2}-w_{1}, w_{2}-z_{2 r}\right\rangle\right) /\left(\left\|w_{2}-z_{2 r}\right\|^{2}\right)\right)$

and

$$
\begin{array}{r}
\left(\left\langle w_{2}-w_{1}, w_{2}-z_{2 r}\right\rangle\right) /\left(\left\|w_{2}-z_{2 r}\right\|^{2}\right) \\
=\left(B-\left\langle w_{2}, z_{2 r}\right\rangle-C+\left\langle w_{1}, z_{2 r}\right\rangle\right) / \\
\left(B+\left\langle z_{2 r}, z_{2 r}\right\rangle-2\left\langle w_{2}, z_{2 r}\right\rangle\right)
\end{array}
$$

[using (60)-(62)]; hence $a_{i}^{\text {new }}=q_{2} b_{i}+\left(1-q_{2}\right) a_{i}, i \in$ $I_{2}$. Continue with step 2).

This algorithm (RCH-SK) has almost the same complexity as the Schlesinger-Kozinec (SK) one (the extra cost is the sort involved in each step to find the least $\left\lceil 1 / \mu_{1}\right\rceil$ and $\left\lceil 1 / \mu_{2}\right\rceil$ inner 
TABLE I

COMPARATIVE RESUlts FOR THE SMO ALgORITHM [11] WiTH THE ALGORITHM PRESENTED IN THIS WORK (RCH-SK)

\begin{tabular}{|ccrrr|r|}
\hline Method & Dataset & $\begin{array}{r}\text { Time } \\
(\mathbf{s e c})\end{array}$ & $\begin{array}{r}\text { Kernel } \\
\text { evaluations }\end{array}$ & $\begin{array}{r}\text { Success } \\
\text { Rate (\%) }\end{array}$ \\
\hline \hline SMO & \multirow{2}{*}{ Checkerboard } & 2276 & 84496594 & 88.17 \\
RCH-SK & & $\mathbf{6 1 8}$ & $\mathbf{2 8 9 1 6 6 3 2}$ & $\mathbf{9 0 . 1 3}$ \\
\hline SMO & \multirow{2}{*}{ Diabetes } & 811 & 1945488 & $\mathbf{7 6 . 6 6}$ \\
RCH-SK & & $\mathbf{1 8 0}$ & $\mathbf{1 9 0 2 7 3 5}$ & 76.20 \\
\hline
\end{tabular}

products, plus the cost to evaluate the inner product $\left\langle z_{r}, z_{r}\right\rangle$ ); the same caching scheme can be used, with only $O\left(\left|I_{1}\right|+\left|I_{2}\right|\right)$ storage requirements.

\section{RESULTS}

In the sequel, some representative results of RCH-SK algorithm are included, concerning two known nonseparable datasets, since the separable cases work in exactly the same way as the SK algorithm, proposed in [16]. Two datasets were chosen. One is an artificial dataset of a two-dimensional (2-D) checkerboard with 800 training points in $4 \times 4$ cells, similar to the dataset found in [25]. The reason that a 2-D example was chosen is to make possible the graphical representation of the results. The second dataset is the Pima Indians Diabetes dataset, with 768 eight-dimensional (8-D) training patterns [26]. Each dataset was trained to achieve comparable success rates for both algorithms, the one presented here (RCH-SK) and the SMO algorithm presented in [11], using the same model (kernel parameters). The results of both algorithms (total run time and number of kernel evaluations) were compared and summarized in Table I. An Intel Pentium M PC has been used for the tests.

1) Checkerboard: A set of 800 (Class A: 400, Class B: 400) randomly generated points on a 2-D checkerboard of $4 \times 4$ cells was used. Each sample attribute ranged from -4 to 4 and the margin was -0.1 (the negative value indicating the overlapping between classes, i.e., the overlapping of the cells). A RBF kernel was used with $\sigma=0.9$ and the success rate was estimated using 40 -fold cross validation (40 randomly generated partitions of 20 samples each, the same for both algorithms). The classification results of both methods are shown in Fig. 8.

2) Diabetes: The 8-D 768 samples dataset was used to train both classifiers. The model (RBF kernel with $\sigma=20$ ), as well as the error rate estimation procedure (cross validation on 100 realizations of the samples) that was used for both algorithms, is found in [26]. Both classifiers (SMO and RCH-SK) closely approximated the success rate $76.47 \%( \pm 1.73)$, reported in [26].

As it is apparent from Table I, substantial reductions with respect to run-time and kernel evaluations can be achieved using the new geometric algorithm (RCH-SK) proposed here. These results indicate that exploiting the theorems and propositions presented in this paper can lead to geometric algorithms that can be considered as viable alternatives to already known decomposition schemes.
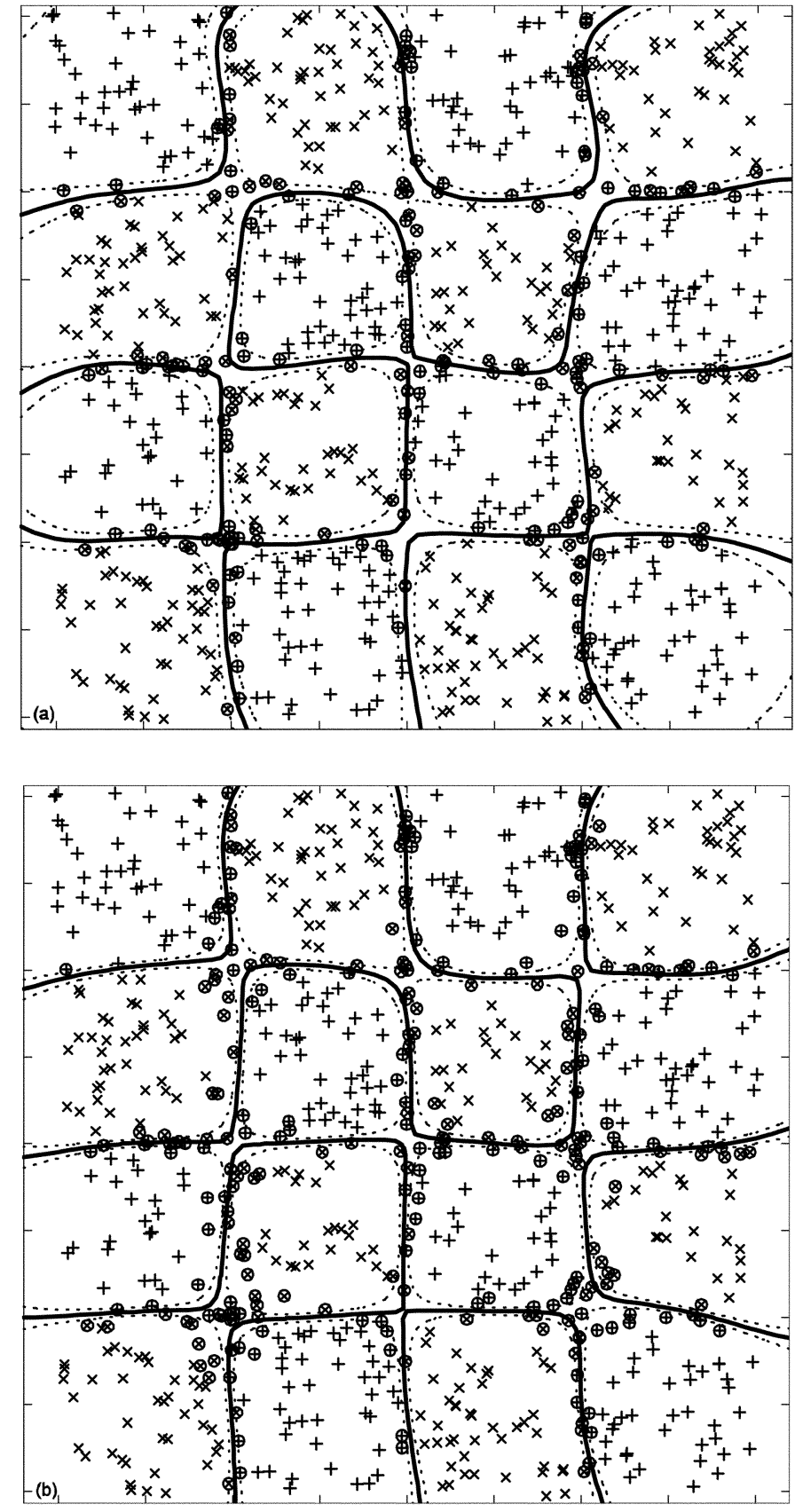

Fig. 8. Classification results for the checkerboard dataset for (a) SMO and (b) RCH-SK algorithms. Circled points are support vectors.

\section{CONCLUSION}

The SVM approach to machine learning is known to have both theoretical and practical advantages. Among these are the sound mathematical foundation of SVM (supporting their generalization bounds and their guaranteed convergence to the global optimum unique solution), their overcoming of the "curse of dimensionality" (through the "kernel trick"), and the intuition they display. The geometric intuition is intrinsic to the structure of SVM and has found application in solving both the separable and nonseparable problem. The iterative geometric algorithm of Schlesinger and Kozinec, modified here to work for the nonseparable task employing RCHs, resulted in a very promising method of solving SVM. The algorithm 
presented here does not use any heuristics and provides a clear understanding of the convergence process and the role of the parameters used. Furthermore, the penalty factor $\mu$ (which has clear meaning corresponding to the reduction factor of each convex hull) can be set different for each class, reflecting the importance of each class.

\section{APPENDIX}

Proof of Lemma 1: In case that $\mu=1$ the lemma is obviously true since $S=\{0,1\}$.

The other case will be proved by contradiction; so, let $0<$ $\mu<1$ and $w \in \mathrm{R}(X, \mu)$ be a point of this $\mathrm{RCH}$. Furthermore, suppose that $w$ is a reduced convex combination with $\mathrm{M}$ the number of coefficients for which $a_{i}=\mu, \Lambda$ the number of coefficients for which $a_{i}=1-\lfloor 1 / \mu\rfloor \mu$ and $r$ the position of the only coefficient of $w$ such that $a_{r} \notin S$ with

$$
0<a_{r}<\mu
$$

by assumption.

Clearly, $\mathrm{M}+\Lambda+1 \leqslant k \Rightarrow \mathrm{M}+\Lambda<k$. Since $0<\mu<1$, it is $1 / \mu>1 \Rightarrow\lfloor 1 / \mu\rfloor \geqslant 1$. Besides, it is

$$
\lfloor 1 / \mu\rfloor \leqslant 1 / \mu \leqslant\lfloor 1 / \mu\rfloor+1 .
$$

From the first inequality of (23) it is $\lfloor 1 / \mu\rfloor \mu \leqslant 1 \Leftrightarrow 1-$ $\lfloor 1 / \mu\rfloor \mu \geqslant 0$ and from the second inequality of (23) it is $1-$ $\lfloor 1 / \mu\rfloor \mu<\mu$. These inequalities combined become

$$
0 \leqslant 1-\lfloor 1 / \mu\rfloor \mu<\mu
$$

According to the above and since $\sum_{i=1}^{k} a_{i}=1$, it is

$$
\mathrm{M} \mu+\Lambda(1-\lfloor 1 / \mu\rfloor \mu)+a_{r}=1 .
$$

Two distinct cases need to be examined: 1$)\lfloor 1 / \mu\rfloor=1 / \mu$ and 2) $\lfloor 1 / \mu\rfloor<1 / \mu$.

1) Let

$$
\lfloor 1 / \mu\rfloor=1 / \mu .
$$

Then $\lfloor 1 / \mu\rfloor \mu=1$ and

$$
1-\lfloor 1 / \mu\rfloor \mu=0 .
$$

Substituting the above to (25), it becomes $\mathrm{M} \mu+a_{r}=1$ and, therefore

$$
\mathrm{M} \mu=1-a_{r} .
$$

a) If $\mathrm{M}=\lfloor 1 / \mu\rfloor$ then $\mathrm{M} \mu=\lfloor 1 / \mu\rfloor \mu$, which, substituted into (28) and using (27), gives $a_{r}=0$, which contradicts to the assumption that $0<a_{r}$.

b) If $\mathrm{M}>\lfloor 1 / \mu\rfloor$ then $\mathrm{M} \mu>\lfloor 1 / \mu\rfloor \mu$ and from (26) it gives $\mathrm{M} \mu>1$ which is a contradiction.

c) If $\mathrm{M}<\lfloor 1 / \mu\rfloor$ then

$$
(\mathrm{M}+1) \mu \leqslant\lfloor 1 / \mu\rfloor \mu, \quad \text { or }(\mathrm{M}+1) \mu \leqslant 1 .
$$

But since $\mu>a_{r} \Rightarrow \mu-a_{r}>0$, which through (28) gives $\mu-1+\mathrm{M} \mu>0$ or $(\mathrm{M}+1) \mu>1$, a contradiction to (29).

2) Let

$$
\lfloor 1 / \mu\rfloor<1 / \mu \text {. }
$$

Then

$$
1-\lfloor 1 / \mu\rfloor \mu>0
$$

and

$$
\lfloor 1 / \mu\rfloor \mu<1 .
$$

The cases when $\Lambda=1$ and $\Lambda>1$ will be considered separately.

a) Let

$$
\Lambda=1
$$

which, substituted to (25) gives

$\mathrm{M} \mu+1-\lfloor 1 / \mu\rfloor \mu+a_{r}=1 \Rightarrow a_{r}=\lfloor 1 / \mu\rfloor \mu-\mathrm{M} \mu$.

i) Let $\mathrm{M}=\lfloor 1 / \mu\rfloor$; consequently (34) gives by substitution $a_{r}=\lfloor 1 / \mu\rfloor \mu-\lfloor 1 / \mu\rfloor \mu=0$ which is a contradiction.

ii) Let $\mathrm{M}>\lfloor 1 / \mu\rfloor \Rightarrow \mathrm{M} \mu>\lfloor 1 / \mu\rfloor \mu$; substituting this value in (34) gives $a_{r}<0$ which is a contradiction.

iii) Let $\mathrm{M}<\lfloor 1 / \mu\rfloor \Rightarrow(\mathrm{M}+1) \mu \leqslant\lfloor 1 / \mu\rfloor \mu$ and, using (34), gives $a_{r} \geqslant(\mathrm{M}+1) \mu-\mathrm{M} \mu \Rightarrow a_{r} \geqslant$ $\mu$ which is a contradiction.

b) Let

$$
\Lambda>1 .
$$

i) If $\mathrm{M}=\lfloor 1 / \mu\rfloor$ then, setting $x \equiv 1-\lfloor 1 / \mu\rfloor \mu$ and observing that $x>0$ from (31), (25) becomes $x(1-\Lambda)=a_{r}$ which is a contradiction, since the LHS is negative whereas the RHS is positive.

ii) Similarly, if $M>\lfloor 1 / \mu\rfloor$ then

$$
\mathrm{M} \mu>\lfloor 1 / \mu\rfloor \mu .
$$

Setting $x \equiv 1-\lfloor 1 / \mu\rfloor \mu$ and observing from (31) that $x>0$, (25) through (36) becomes $x(1-\Lambda)>a_{r}$ which is a contradiction, since the LHS is negative whereas the RHS is positive.

iii) If $\mathrm{M}<\lfloor 1 / \mu\rfloor$ then there exists a positive integer $\mathrm{K} \geqslant 1$ such that

$$
\mathrm{M}+\mathrm{K}=\lfloor 1 / \mu\rfloor \Rightarrow \mathrm{M} \mu+\mathrm{K} \mu=\lfloor 1 / \mu\rfloor \mu .
$$

This relation, through (25), becomes

$$
\begin{aligned}
1-\Lambda(1-\lfloor 1 / \mu\rfloor \mu)-a_{r}+\mathrm{K} \mu & =\lfloor 1 / \mu\rfloor \mu \Rightarrow \\
& a_{r}=\mathrm{K} \mu-(\Lambda-1)(1-\lfloor 1 / \mu\rfloor \mu) .
\end{aligned}
$$


Substituting (38) into (25) gives

$$
\begin{array}{r}
\mathrm{M} \mu+\Lambda(1-\lfloor 1 / \mu\rfloor \mu)+\mathrm{K} \mu-(\Lambda-1)(1-\lfloor 1 / \mu\rfloor \mu)=1 \Rightarrow \\
(\mathrm{M}+\mathrm{K}) \mu+1-\lfloor 1 / \mu\rfloor \mu=1 .
\end{array}
$$

This last relation states that, in this case, there is an alternative configuration to construct $w$ [other than (25)], which does not contain the coefficient $a_{r}$ but only coefficients belonging to the set $S$. This contradicts to the initial assumption that there exists a point $w$ in a $\mathrm{RCH}$ that is a reduced convex combination of points of $X$ with all except one $\left(a_{r}\right)$ coefficients belonging to $S$, since $a_{r}$ is not necessary to construct $w$.

Therefore, the lemma has been proved.

Proof of Lemma 2: Let $0<\mu<\lambda, w_{1}=\lambda s_{i_{1}}+$ $\mu \sum_{m=2}^{k+1} s_{i_{m}}$, where $s_{i_{p}} \leqslant s_{i_{q}}$ if $p<q$ and $w_{2}=$ $\lambda s_{j_{1}}+\mu \sum_{m=2}^{k+1} s_{j_{m}}$, where no ordering is imposed on the $s_{j_{m}}$. It is certain that $s_{i_{1}} \leqslant s_{j_{1}} \Rightarrow \lambda s_{i_{1}} \leqslant \lambda s_{j_{1}}$. $\sum_{m=2}^{k+1} s_{j_{m}}$ is minimum if the $k$ additives are the $k$ minimum elements of $S$. If $s_{i_{1}}=s_{j_{1}}$ the proof is trivial. Therefore, let $s_{i_{1}} \neq s_{j_{1}}$ and hence $i_{1} \neq j_{1}$. Thus $i_{1} \in\left\{j_{2}, \ldots, j_{k+1}\right\}$ and $j_{1} \in\left\{i_{2}, \ldots, i_{k+1}\right\}$. In general, let $i_{1}=j_{p}$ and $i_{p}=j_{1}$, with $j_{m}=i_{m}$, where $m \in V \equiv\{2, \ldots, k+1\}-\{p\}$. Then $w_{1}-w_{2}=\lambda s_{i_{1}}+\mu s_{i_{p}}+\mu \sum_{m \in V} s_{i_{m}}-\lambda s_{j_{1}}-$ $\mu s_{j_{p}}-\mu \sum_{m \in V} s_{j_{m}}=\lambda s_{i_{1}}+\mu s_{i_{p}}-\lambda s_{i_{p}}-\mu s_{i_{1}}=$ $\lambda\left(s_{i_{1}}-s_{i_{p}}\right)-\mu\left(s_{i_{1}}-s_{i_{p}}\right)=(\lambda-\mu)\left(s_{i_{1}}-s_{i_{p}}\right) \leqslant 0$; equality is valid only if $s_{i_{1}}=\cdots=s_{i_{p}}$.

Each of the remaining cases (i.e., $\lambda=0$, or $0<\lambda<\mu$ ) is proved similarly as above.

Calculation of the Intermediate Quantities Involved in the Algorithm: For the calculation of $\left\|w_{1}-w_{2}\right\|$, it is

$$
\left\|w_{1}-w_{2}\right\|=\sqrt{\left\langle w_{1}, w_{1}\right\rangle+\left\langle w_{2}, w_{2}\right\rangle-2\left\langle w_{1}, w_{2}\right\rangle} .
$$

Setting

$$
\begin{aligned}
\left\langle w_{1}, w_{1}\right\rangle & =\left\langle\sum_{i \in I_{1}} a_{i} x_{i}, \sum_{j \in I_{1}} a_{j} x_{j}\right\rangle \\
& =\sum_{i \in I_{1}} \sum_{j \in I_{1}} a_{i} a_{j}\left\langle x_{i}, x_{j}\right\rangle \equiv A \\
\left\langle w_{2}, w_{2}\right\rangle & =\left\langle\sum_{i \in I_{2}} a_{i} x_{i}, \sum_{j \in I_{2}} a_{j} x_{j}\right\rangle \\
& =\sum_{i \in I_{2}} \sum_{j \in I_{2}} a_{i} a_{j}\left\langle x_{i}, x_{j}\right\rangle \equiv B
\end{aligned}
$$

and

$$
\begin{aligned}
\left\langle w_{1}, w_{2}\right\rangle & =\left\langle\sum_{i \in I_{1}} a_{i} x_{i}, \sum_{j \in I_{2}} a_{j} x_{j}\right\rangle \\
& =\sum_{i \in I_{1}} \sum_{j \in I_{2}} a_{i} a_{j}\left\langle x_{i}, x_{j}\right\rangle \equiv C
\end{aligned}
$$

it is

$$
\left\|w_{1}-w_{2}\right\|=\sqrt{A+B-2 C} .
$$

According to the above Proposition 3, any extreme point of the RCHs has the form

$$
z_{r}=\left\{\begin{array}{rl}
z_{1 r}=\sum_{i \in I_{1}} b_{i} x_{i}, & \\
b_{i} \in\left\{0, \lambda_{1}, \mu_{1}\right\}, & \sum_{i \in I_{1}} b_{i}=1 \\
z_{2 r}=\sum_{i \in I_{2}} b_{i} x_{i}, & \\
b_{i} \in\left\{0, \lambda_{2}, \mu_{2}\right\}, & \sum_{i \in I_{2}} b_{i}=1
\end{array} .\right.
$$

The projection of $z_{r}$ onto the direction $w_{1}-w_{2}$ is needed. According to Theorem 2 , the minimum of this projection is formed as the weighted sum of the projections of the original points onto the direction $w_{1}-w_{2}$.

Specifically, the projection of $z_{1 r}$ onto $w_{1}-w_{2}$, where $z_{1 r} \in \mathrm{R}\left(X_{1}, \mu_{1}\right)$ and $z_{1 r}=\sum_{i \in I_{1}} b_{i} x_{i}, b_{i} \in$ $\left\{0, \lambda_{1}, \mu_{1}\right\}, \sum_{i \in I_{1}} b_{i}=1$, is $p\left(z_{1 r}\right)=\left\langle z_{1 r}, w_{1}-w_{2}\right\rangle /$ $\left\|w_{1}-w_{2}\right\|$, and by (44)

$$
p\left(z_{1 r}\right)=\left\langle z_{1 r}, w_{1}-w_{2}\right\rangle /(\sqrt{A+B-2 C}) .
$$

Since the quantity $\sqrt{A+B-2 C}$ is constant at each step, for the calculation of $\min \left(p\left(z_{1 r}\right)\right)$, the ordered inner products of $x_{i}, x_{i} \in I_{1}$, with $w_{1}-w_{2}$ must be formed. From them, the smallest $\left\lceil 1 / \mu_{1}\right\rceil$ numbers, each multiplied by the corresponding coefficient (as of Theorem 2), must be summed. Therefore, using $\lambda_{1} \equiv 1-\left\lfloor 1 / \mu_{1}\right\rfloor \mu_{1}, m_{1} \equiv\left\lfloor 1 / \mu_{1}\right\rfloor, \lambda_{2} \equiv 1-\left\lfloor 1 / \mu_{2}\right\rfloor \mu_{2}$, $m_{2} \equiv\left\lfloor 1 / \mu_{2}\right\rfloor$ it is

$$
\begin{aligned}
\left\langle x_{r}, w_{1}\right\rangle & =\left\langle x_{r}, \sum_{i \in I_{1}} a_{i} x_{i}\right\rangle \\
& =\sum_{i \in I_{1}} a_{i}\left\langle x_{i}, x_{r}\right\rangle \equiv D_{r} \\
\left\langle x_{r}, w_{2}\right\rangle & =\left\langle x_{r}, \sum_{i \in I_{2}} a_{i} x_{i}\right\rangle \\
& =\sum_{i \in I_{2}} a_{i}\left\langle x_{i}, x_{r}\right\rangle \equiv E_{r} .
\end{aligned}
$$

In the sequel, the numbers $D_{r}$ must be ordered, for each set of indices, separately

$$
\begin{aligned}
& \tilde{D}_{r}^{1} \equiv\left\{D_{r_{1}}^{1}, \ldots, D_{r_{m_{1}}}^{1}\right\}, \quad r \in I_{1}, \quad i<j \Rightarrow D_{r_{i}}^{1} \leqslant D_{r_{j}}^{1} \\
& \tilde{D}_{r}^{2} \equiv\left\{D_{r_{1}}^{2}, \ldots, D_{r_{m_{2}}}^{2}\right\}, \quad r \in I_{2}, \quad i<j \Rightarrow D_{r_{i}}^{2} \leqslant D_{r_{j}}^{2}
\end{aligned}
$$

and

$$
\begin{aligned}
& \tilde{E}_{r}^{1} \equiv\left\{E_{r_{1}}^{1}, \ldots, E_{r_{m_{1}}}^{1}\right\}, \quad r \in I_{1}, \quad i<j \Rightarrow E_{r_{i}}^{1} \leqslant E_{r_{j}}^{1} \\
& \tilde{E}_{r}^{2} \equiv\left\{E_{r_{1}}^{2}, \ldots, E_{r_{m_{2}}}^{2}\right\}, \quad r \in I_{2}, \quad i<j \Rightarrow E_{r_{i}}^{2} \leqslant E_{r_{j}}^{2}
\end{aligned}
$$

With the above definitions [(47)-(52)] and applying Theorem 2 , it is $\min \left(\left\langle z_{1 r}, w_{1}-w_{2}\right\rangle\right)=\mu_{1}\left(\sum_{j=1}^{m_{1}}\left(\tilde{D}_{r_{j}}^{1}-\tilde{E}_{r_{j}}^{1}\right)\right)$ $+\lambda_{1}\left(\tilde{D}_{r_{m_{1}+1}}^{1}-\tilde{E}_{r_{m_{1}+1}}^{1}\right)$ and consequently [using definitions 


$$
\begin{aligned}
\min \left(m\left(z_{1 r}\right)\right) & =\frac{\mu_{1} \sum_{j=1}^{m_{1}} \tilde{D}_{r_{j}}^{1}+\lambda_{1} \tilde{D}_{r_{m_{1}+1}}^{1}-\mu_{1} \sum_{j=1}^{m_{1}} \tilde{E}_{r_{j}}^{1}-\lambda_{1} \tilde{E}_{r_{m_{1}+1}}^{1}-C+B}{\sqrt{A+B-2 C}} \\
\min \left(m\left(z_{2 r}\right)\right) & =\frac{\mu_{2} \sum_{j=1}^{m_{2}} \tilde{E}_{r_{j}}^{2}+\lambda_{2} \tilde{E}_{r_{m_{2}+1}}^{2}-\mu_{2} \sum_{j=1}^{m_{2}} \tilde{D}_{r_{j}}^{2}-\lambda_{2} \tilde{D}_{r_{m_{2}+1}}^{2}-C+A}{\sqrt{A+B-2 C}} .
\end{aligned}
$$

(20), (40)--(43) and (47)-(52)], respectively, (53) and (54), as shown at the top of the page.

Finally, for the adaptation phase, the scalar quantities

$$
\frac{\left\langle w_{1}-w_{2}, w_{1}-z_{1 r}\right\rangle}{\left\|w_{1}-z_{1 r}\right\|^{2}}=\frac{A-\left\langle w_{1}, z_{1 r}\right\rangle-C+\left\langle w_{2}, z_{1 r}\right\rangle}{A+\left\langle z_{1 r}, z_{1 r}\right\rangle-2\left\langle w_{1}, z_{1 r}\right\rangle}
$$

and

$$
\frac{\left\langle w_{2}-w_{1}, w_{2}-z_{2 r}\right\rangle}{\left\|w_{2}-z_{2 r}\right\|^{2}}=\frac{B-\left\langle w_{2}, z_{2 r}\right\rangle-C+\left\langle w_{1}, z_{2 r}\right\rangle}{B+\left\langle z_{2 r}, z_{2 r}\right\rangle-2\left\langle w_{2}, z_{2 r}\right\rangle}
$$

are needed in the calculation of $q$. Therefore, the inner products $\left\langle w_{1}, z_{1 r}\right\rangle,\left\langle w_{2}, z_{1 r}\right\rangle,\left\langle z_{1 r}, z_{1 r}\right\rangle,\left\langle w_{1}, z_{2 r}\right\rangle,\left\langle w_{2}, z_{2 r}\right\rangle$ and $\left\langle z_{2 r}, z_{2 r}\right\rangle$ need to be calculated. The result is

$$
\begin{aligned}
\left\langle w_{1}, z_{1 r}\right\rangle= & \mu_{1} \sum_{j=1}^{m_{1}} \tilde{D}_{r_{j}}^{1}+\lambda_{1} \tilde{D}_{r_{m_{1}+1}}^{1} \\
\left\langle w_{2}, z_{1 r}\right\rangle= & \mu_{1} \sum_{j=1}^{m_{1}} \tilde{E}_{r_{j}}^{1}+\lambda_{1} \tilde{E}_{r_{m_{1}+1}}^{1} \\
\left\langle z_{1 r}, z_{1 r}\right\rangle= & \left(\mu_{1}\right)^{2} \sum_{j=1}^{m_{1}} \sum_{i=1}^{m_{1}}\left\langle x_{r_{j}}, x_{r_{i}}\right\rangle \\
& +\left(\lambda_{1}\right)^{2}\left\langle x_{r_{m_{1}+1}}, x_{r_{m_{1}+1}}\right\rangle \\
& +2 \lambda_{1} \mu_{1} \sum_{j=1}^{m_{1}}\left\langle x_{r_{m_{1}+1}}, x_{r_{j}}\right\rangle \\
\left\langle w_{1}, z_{2 r}\right\rangle= & \mu_{2} \sum_{j=1}^{m_{2}} \tilde{D}_{r_{j}}^{2}+\lambda_{2} \tilde{D}_{r_{m_{2}+1}}^{2} \\
\left\langle w_{2}, z_{2 r}\right\rangle= & \mu_{2} \sum_{j=1}^{m_{2}} \tilde{E}_{r_{j}}^{2}+\lambda_{2} \tilde{E}_{r_{m_{2}+1}}^{2} \\
\left\langle z_{2 r}, z_{2 r}\right\rangle= & \left(\mu_{2}\right)^{2} \sum_{j=1}^{m_{2}} \sum_{i=1}^{m_{2}}\left\langle x_{r_{j}}, x_{r_{i}}\right\rangle \\
& +\left(\lambda_{2}\right)^{2}\left\langle x_{r_{m_{2}+1}}, x_{r_{m_{2}+1}}\right\rangle \\
& +2 \lambda_{2} \mu_{2} \sum_{j=1}^{m_{2}}\left\langle x_{r_{m_{2}+1}}, x_{r_{j}}\right\rangle .
\end{aligned}
$$

\section{REFERENCES}

[1] N. Cristianini and J. Shawe-Taylor, An Introduction to Support Vector Machines and Other Kernel-Based Learning Methods. Cambridge, U.K.: Cambridge Univ. Press, 2000.

[2] S. Theodoridis and K. Koutroumbas, Pattern Recognition, 2nd ed. New York: Academic Press, 2003.

[3] C. Cortes and V. N. Vapnik, "Support Vector Networks," Mach. Learn., vol. 20, no. 3, pp. 273-297, Sep. 1995.

[4] I. El-Naqa, Y. Yang, M. Wernik, N. Galatsanos, and R. Nishikawa, "A support vector machine approach for detection of microcalsifications," IEEE Trans. Med. Imag., vol. 21, no. 12, pp. 1552-1563, Dec. 2002.
[5] T. Joachims, "Text categorization with support vector machines: Learning with many relevant features," in Proc. 10th European Conf. Machine Learning (ECML), Chemnitz, Germany, 1998, pp. 137-142.

[6] E. Osuna, R. Freund, and F. Girosi, "Training support vector machines: An application to face detection," in Proc. IEEE Conf. Computer Vision and Pattern Recognition, 1997, pp. 130-136.

[7] M. P. S. Brown, W. N. Grundy, D. Lin, N. Cristianini, C. W. Sugnet, T. S. Furey, M. Ares Jr., and D. Haussler, "Knowledge-based analysis of microarray gene expression data by using support vector machines," in Proc. Nat. Acad. Sci. 97, 2000, pp. 262-267.

[8] A. Navia-Vasquez, F. Perez-Cuz, and A. Artes-Rodriguez, "Weighted least squares training of support vector classifiers leading to compact and adaptive schemes," IEEE Trans. Neural Netw., vol. 12, no. 5, pp. 1047-1059, Sep. 2001.

[9] D. J. Sebald and J. A. Buklew, "Support vector machine techniques for nonlinear equalization," IEEE Trans. Signal Process., vol. 48, no. 11, pp. 3217-3227, Nov. 2000

[10] D. Zhou, B. Xiao, H. Zhou, and R. Dai, "Global Geometry of SVM Classifiers," Institute of Automation, Chinese Academy of Sciences, Tech. Rep. AI Lab., 2002. Submitted to NIPS.

[11] J. Platt, "Fast training of support vector machines using sequential minimal optimization," in Advances in Kernel Methods-Support Vector Learning, B. Schölkopf, C. Burges, and A. Smola, Eds. Cambridge, MA: MIT Press, 1999, pp. 185-208.

[12] K. P. Bennett and E. J. Bredensteiner, "Geometry in learning," in Geometry at Work, C. Gorini, E. Hart, W. Meyer, and T. Phillips, Eds. Washington, DC: Mathematical Association of America, 1998.

[13] — "Duality and Geometry in SVM classifiers," in Proc. 17th Int. Conf. Machine Learning, P. Langley, Ed.. San Mateo, CA, 2000, pp. 57-64.

[14] D. J. Crisp and C. J. C. Burges, "A geometric interpretation of $\nu$-SVM classifiers," Adv. Neural Inform. Process. Syst. (NIPS) 12, pp. 244-250, 1999.

[15] S. S. Keerthi, S. K. Shevade, C. Bhattacharyya, and K. R. K. Murthy, "A fast iterative nearest point algorithm for support vector machine classifier design,” Dept. CSA, IISc, Bangalore, Karnataka, India, Tech. Rep. TR-ISL-99-03, 1999.

[16] V. Franc and V. Hlaváč, "An iterative algorithm learning the maximal margin classifier," Pattern Recognit., vol. 36, no. 9, pp. 1985-1996, 2003.

[17] T. T. Friess and R. Harisson, "Support vector neural networks: the kernel adatron with bias and soft margin," Univ. Sheffield, Dept. ACSE, Tech. Rep. ACSE-TR-752, 1998.

[18] B. Schölkopf and A. Smola, Learning with Kernels-Support Vector Machines, Regularization, Optimization and Beyond. Cambridge, MA: MIT Press, 2002.

[19] V. N. Vapnik, Statistical Learning Theory. New York: Wiley, 1998.

[20] D. G. Luenberger, Optimization by Vector Space Methods. New York: Wiley, 1969.

[21] R. T. Rockafellar, Convex Analysis. Princeton, NJ: Princeton Univ. Press, 1970.

[22] S. G. Nash and A. Sofer, Linear and Nonlinear Programming. New York: McGraw-Hill, 1994.

[23] C. J. C. Burges, "A tutorial on support vector machines for pattern recognition," Data Mining and Knowledge Discovery, vol. 2, no. 2, pp. 121-167, 1998.

[24] J.-B. Hiriart-Urruty and C. Lemaréchal, Convex Analysis and Minimization Algorithms I. New York: Springer-Verlag, 1991.

[25] L. Kaufman, "Solving the quadratic programming problem arising in support vector classification," in Advances in Kernel Methods-Support Vector Learning, B. Schölkopf, C. Burges, and A. Smola, Eds. Cambridge, MA: MIT Press, 1999, pp. 147-167.

[26] G. Rätsch, T. Onoda, and K.-R. Müller, "Soft margins for AdaBoost," in Machine Learning. Norwell, MA: Kluwer, 2000, vol. 42, pp. 287-320.

Michael E. Mavroforakis photograph and biography not available at the time of publication.

Sergios Theodoridis (M'87-SM'02) photograph and biography not available at the time of publication. 